\section{Filling in the blind spot}

SIR - Andrews and Campbell ${ }^{1}$ described an ingenious experiment on the blind spot ${ }^{2}$. Does 'filling in' of the blind spot involve creating an explicit representation $^{3}$ of the surrounding colour or pattern, or do we simply ignore the absence of signals from this region just as we ignore the back of our head?

To find out, we placed a yellow ring or doughnut (outer diameter $8^{\circ}$; inner diameter $4.5^{\circ}$ ) on the blind spot so that its inner margin fell entirely inside the blind spot. The ring then looked like a homogeneous yellow disk, being 'filled in' by the yellow from the ring. Furthermore, when we had several rings scattered randomly in the visual field, with one ring alone being positioned on the blind spot, then the latter 'popped out'

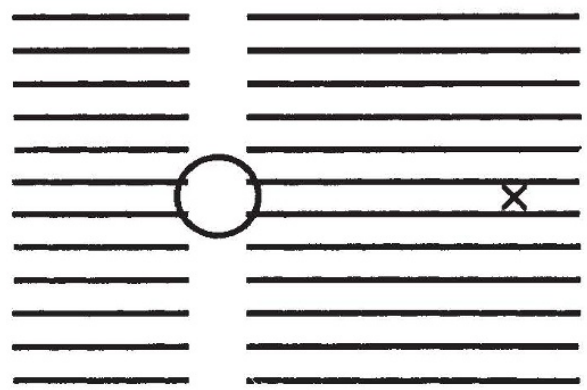

This display schematically iliustrates a vertical illusory strip that passes through the subject's blind spot (circle) as he fixates the $X$ with his left eye. Interestingly, it is the illusory contours that get 'completed' rather than the real horizontal lines that define them.

preattentively even in naive subjects, that is, it was conspicuously visible as a disk against a background of rings. Because pop-out implies early visual processing, we conclude that the filling in also occurs relatively early. Also, our results suggest that one really does 'fill in' the blind spot - people do not just ignore what is out there. For how can something being ignored 'pop-out'?

We also find that filling in is a sensory rather than cognitive process. A line traversing the blind spot is seen as complete but if a circle (diameter $10^{\circ}$ ) falls partially on the blind spot, the visual system does not complete the arc of the circle; it looks 'chopped off'. Hence, filling in occurs at the level of surface and contour interpolation rather than the level of object representation.

Finally, if a vertical illusory strip is made to pass through the blind spot, then the visual system completes the illusory contours across the blind spot instead of completing the horizontal lines that define the illusory contours (see figure). Again, if the visual system were simply ignoring the blind spot, it is hard to see how the observer could make this discrimination.

The recent experiments of Gilbert and Wiesel $^{4}$ support our conclusion that the filling in observed in this display is an active process that occurs early in visual processing ${ }^{3}$. Gilbert and Wiesel destroyed a small patch of retina (in cats and monkeys) and recorded from cells in the corresponding area of visual cortex. These cells were initially silent, of course, but within a few minutes the same cells could be excited by visual stimuli that lay outside the scotoma ${ }^{4}$. Next, instead of destroying retinal receptors, Gilbert and Wiesel repeated these experiments using a display similar to the one we used for generating the artificial scotoma. To their surprise, they found that cells which initially had receptive fields within the square now had receptive fields that were much larger and included regions outside the square ${ }^{4}$. Because these cells were originally responding to stimuli inside the scotoma, perhaps higher brain centres are 'fooled' into thinking that stimuli immediately outside the scotoma are

\section{Apolipoprotein B intermediates}

SIR - We showed that, unlike a conventional secretory protein, the aminoterminal $15 \%$ of apolipoprotein B (apo B) translocates the endoplasmic reticulum membrane via transient, transmembrane intermediates before conversion to the fully translocated form ${ }^{1}$. Because these intermediates are wholly extractable from the bilayer at $\mathrm{pH} 11.5$, we suggested that apo B pauses (stops and restarts) in the aqueous translocation channel at several points, perhaps to allow modification, assembly or regulation before secretion. Pease et al. ${ }^{2}$ disagree with our findings and our model of apo $\mathrm{B}$ biogenesis.

The most important difference is that we find intermediates with progressively longer amino-terminal lengths protected from exogenous proteases which become fully protected over time; Pease et al. find only the latter fully protected forms. They believe our findings are due to over-proteolysis, whereas we believe that they have failed to detect transient intermediates. Thus, we propose that the translocation of apo B proceeds stepwise until it is fully protected, whether it is completely in the lumen or associated with the membrane of the endoplasmic reticulum. Pease et al. believe that apo B is cotranslationally inserted into the inner leaflet of the membrane, bypassing any transmembrane translocational intermediates. These issues might have been simply resolved by an exchange of materials and discussion of protocols which we offered through our correspondence with Nature. now actually inside it and this would explain the perceptual 'filling in' effects that we observed.

Furthermore, these results imply that the classical receptive field may be just the tip of an iceberg. Each cell may have thousands of silent synapses that can be reactivated or inhibited - perhaps in seconds - in response to visual stimulation, this can manifest as dynamic changes in receptive field organization. Studying these changes may provide insights about the neural mechanisms underlying perception.

Department of Psychology,

University of California,

La Jolla

California 92093, USA

Andrews, P. R. \& Campbell, F. W. Nature 353, 308 (1991).

. Brewster, D. Letters on Natural Magic (Murray, London, 1832)

Ramachandran, V. S. \& Gregory, R. L. Nature 350 699-702 (1991)

4. Gilbert, C. \& Wiesel, T. N. Soc. Neurosci. Abstr. 17 1090 (1991).

- See the Letter by C. D. Gilbert and T. N. Wiesel on page 150 of this issue.

A second way to resolve the dispute is to test the two models. One model predicts that translocation of apo B pauses at multiple, specific sequences within the protein, and that such pausing can be dissociated into stopping and restarting, which would simplify detection of the otherwise transient intermediates. The other model predicts that translocation does not pause and hence, sequences with this function do not exist in apo $\mathrm{B}$.

We have now ${ }^{3}$ (1) identified a 'pause transfer' sequence early in apo B which pauses translocation of a defined heterologous chimaeric protein; (2) dissociated that pause into distinct stop and restart steps; (3) demonstrated another pause transfer sequence in its native context later in the protein suggesting that multiple such sequences are involved in the biogenesis of apo $\mathrm{B}$; and (4) described a method to keep a transient transmembrane intermediate stopped for up to 2 hours before restarting its translocation post-translationally, rendering it readily detectable even under the experimental conditions of Pease et al. We believe these data will resolve the controversy and will allow progress in the study of this unusual phenomenon.

STEVEN L. CHUCK VISHWANATH R. LINGAPPA

Department of Physiology

University of California, San Francisco,

California 94143-0444, USA

PEASE ET AL. REPLY - The report by 